\title{
Removal of a foreign body from the back of the orbit
}

\author{
F. J. GURTIS AND D. H. PROVAN \\ East Surrey Hospital, Redhill
}

The removal of deeply-embedded foreign bodies always presents a problem. The following case is reported to illustrate a technique used in a small provincial hospital without the aid of elaborate equipment. The standard $x$-ray screen was used, and the procedure would undoubtedly have been easier if an image intensifier had also been available.

\section{Case report}

A boy aged 13 was brought to the casualty department on May 15, 1969. He had been shooting at a tin with an air-gun and had received a severe blow over the left eye. He was found to have a small round puncture wound immediately below the inner end of the left eyebrow in the trochlear region, with slight swelling of the upper lid and the left bulbar conjunctiva. He was complaining of diplopia and there was limitation of movement of the eye in all directions.

\section{Examination}

Radiography revealed a lead air-gun pellet in the back of the orbit (Fig. $1 a, b$ ).

I (a)

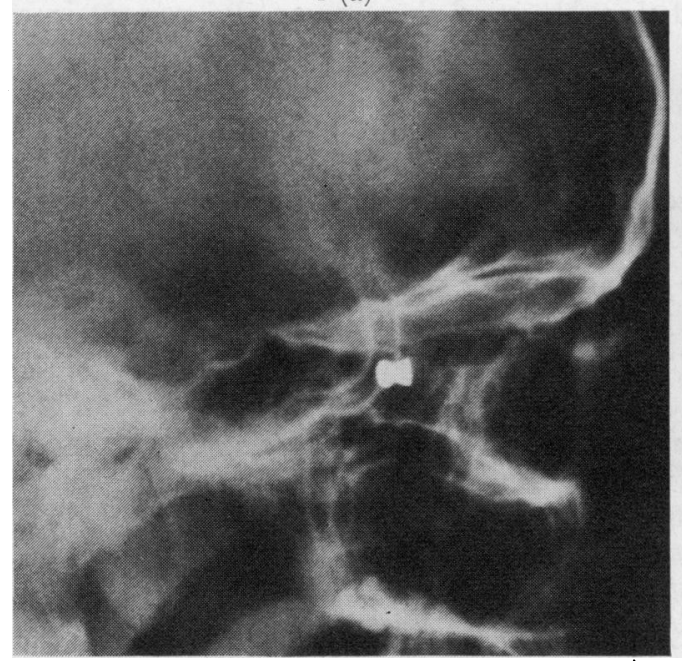

I (b)

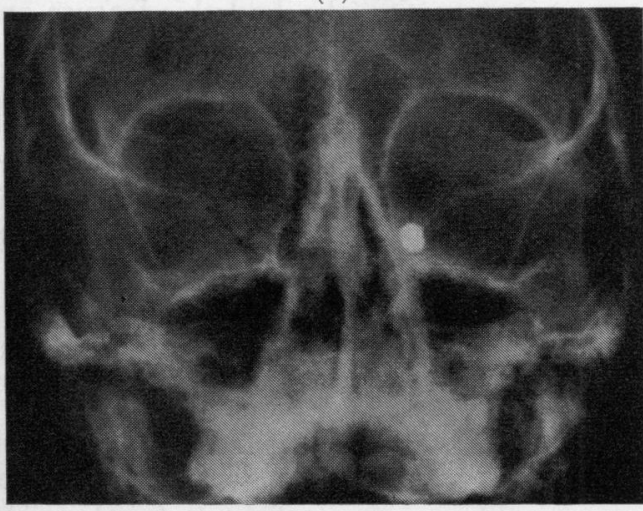

FIG. I graphs

He was admitted to the Eye Unit where the puncture wound was confirmed. By this time there was more marked chemosis and limitation of all movements, with horizontal diplopia. Further localizing $x$ rays revealed that the pellet was lying just nasal to the optic foramen, approximately $5 \mathrm{~cm}$. from the orbital margin (Fig. 2, opposite). 


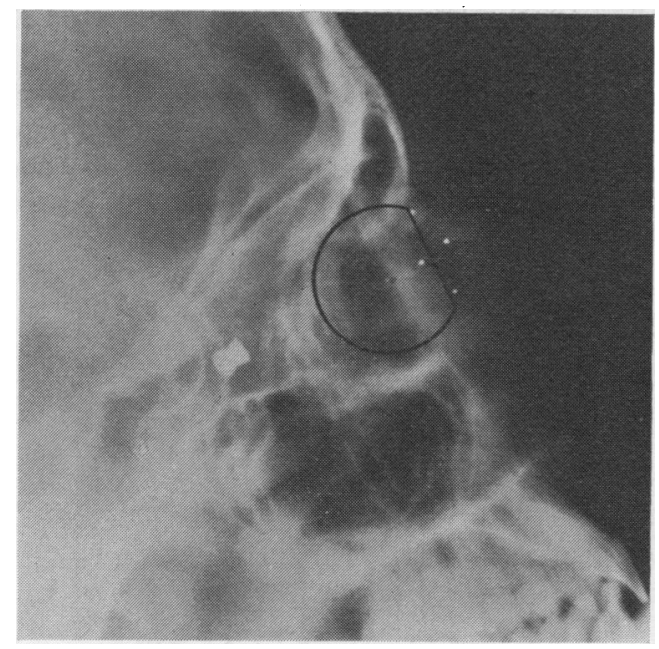

FIG. 2 Localizing radiograph with contact lens in situ. Circle represents size and position of eyeball

\section{Operation}

Under general anaesthesia on the $x$-ray table, using a standard screen, a probe was found to pass easily through the puncture wound and along the track under radiographic guidance until the pellet could be felt. Measurements of depth and direction were confirmed and the probe was replaced by a Hartman's aural crocodile forceps. With similar radiographic guidance the forceps was passed up the track and after very careful feeling it was possible to grip the skirt of the pellet and gradually to withdraw it. It was necessary to enlarge the skin wound slightly in order to extract forceps and pellet together. $2 \mathrm{ml}$. penicillin were then injected along the track and the patient returned to bed.

Postoperatively there was remarkably little reaction, but when the dressing was removed there was found to be total diplopia and complete absence of any adduction of the left eye. Treatment was given with local short-wave therapy.

A Hess screen test (Fig. 3) 4 days later showed complete paralysis of the left medial rectus and secondary overaction of the right lateral rectus. Active duction exercises were given.

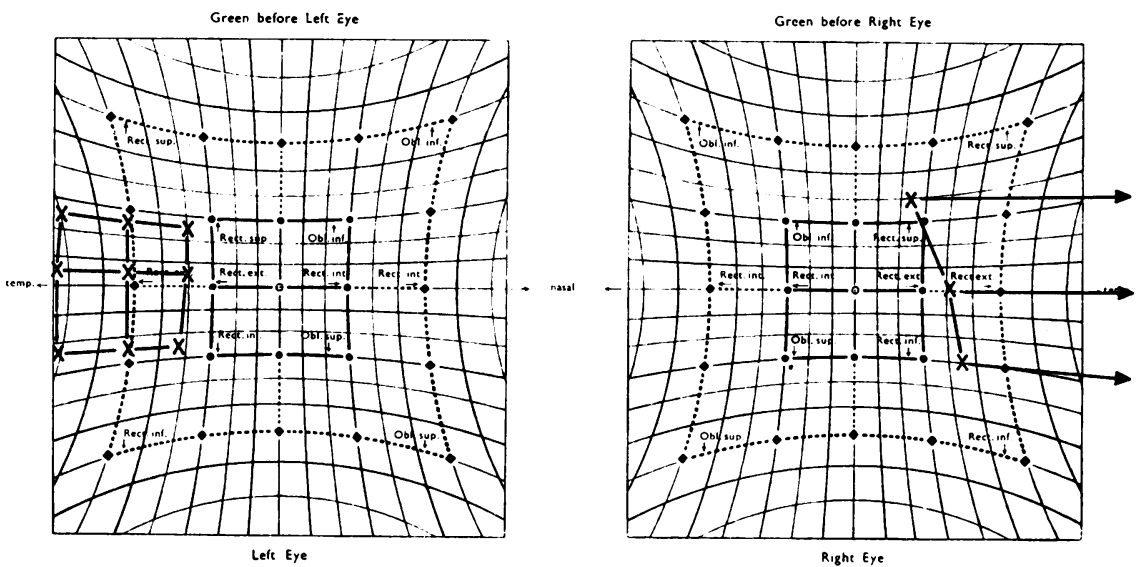

FIG. 3 Hess charts 4 days after removal of foreign body from left orbit

\section{Progress}

On the eighth day he was sent home with a patch for covering each eye alternately. On June 5, i969 the visual acuity in the right eye was $6 / 5$, and in the left $6 / 9$; there was a variable left divergent squint of $20^{\circ}$ to $50^{\circ}$ with no left adduction. He was instructed to persist with duction exercises for 2 minutes 
every half hour, and was allowed to return to school on the understanding that he was to be given rest periods mid-morning and mid-afternoon.

By June 26 he had regained binocular single vision to the left and almost to the straight ahead? position, and was managing to carry on all day at school. The visual acuity had improved to right and left $6 / 4$ and he could move his eye fully in all directions, but it required an effort to maintain $\underset{\vec{\rho}}{\overrightarrow{3}}$ binocular single vision to the right. The Hess screen test (Fig. 4) showed this great improvement.0
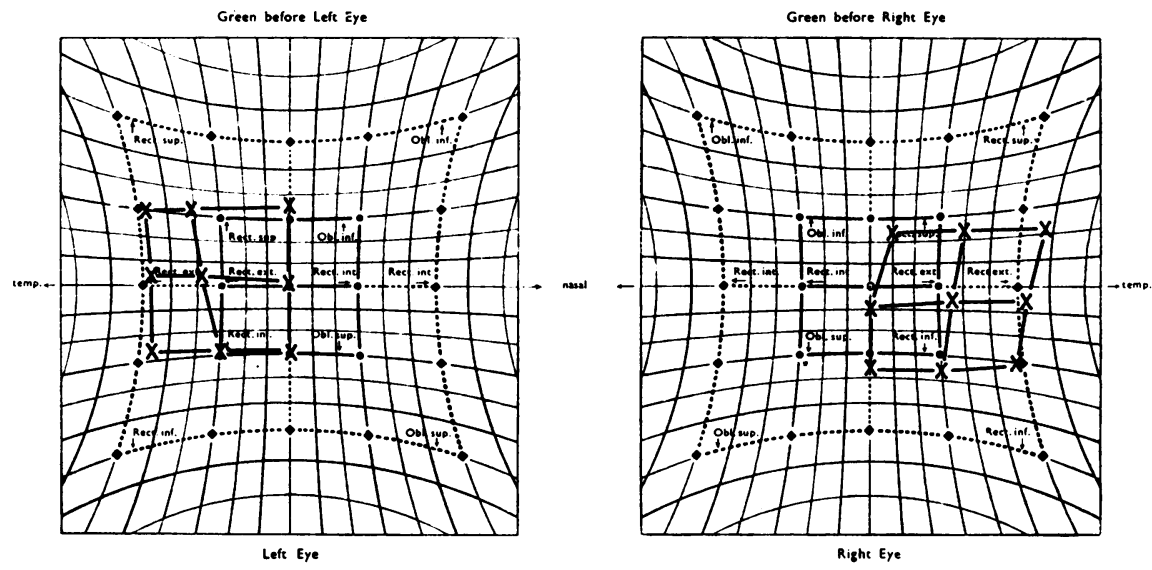

FIG. 4 Hess charts 5 weeks later

Result

On July 24 he had lost all diplopia and the Hess screen test was normal (Fig. 5). The patient noìi has full binocular vision and normal converge
optic nerve damage and he was discharged.

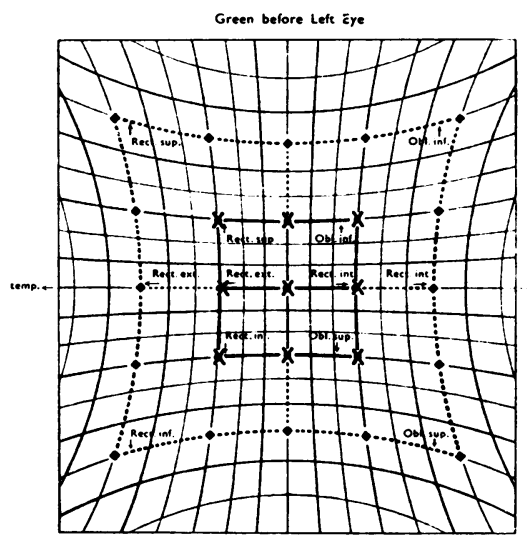

Left Eye

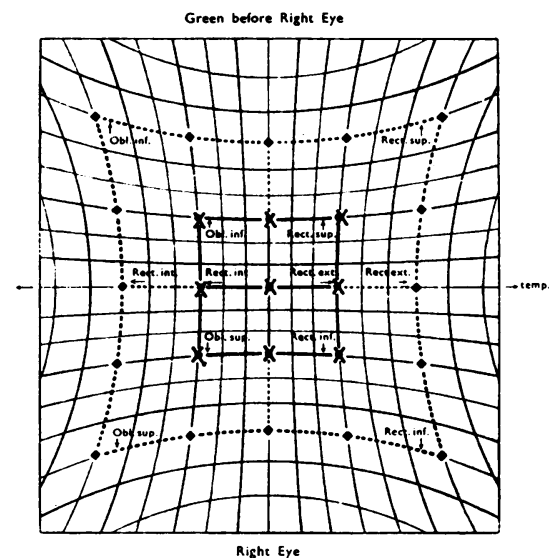

Right Eye

FIG. 5 Hess charts 9 weeks after injury, showing complete recovery of binocular single vision

\section{Comment}

This case is reported in view of the advice in the text books either to leave such a foreign body alone (Stallard, I958), or to attempt to remove it either by a transfrontal approachō or by a lateral orbitotomy (Duke-Elder, 1954). 
It was felt that to leave such a large foreign body so near the apex of the orbit carried a risk of either infection or lead toxic reaction in the neighbourhood of the optic nerve.

The manipulations were carried out with extreme care and gentleness to minimise the risk of further damage and in comparison with the trauma involved by an approach either from above or from the lateral aspect, this method has much to recommend it.

It is a pleasure to record our gratitude to the Radiological Staff and to the Orthoptic Department for their painstaking help and co-operation.

\section{References}

DUKE-ELDER, s. (1954) “Text-book of Ophthalmology", vol. 6, p. 6324. Kimpton, London STAllaRd, H. B. (1958) "Eye Surgery", 3rd ed., p. 824. Wright, Bristol 